\section{Linear-phase fiber lattice equalizer for Gaussian-like spectra}

\author{
Qi Jie Wang ${ }^{a}$, Ying Zhang, MEMBER SPIE ${ }^{B}$, \\ Zhao Gang Dong ${ }^{b}$, and Yeng Chai Soh ${ }^{a}$ \\ ${ }^{a}$ Nanyang Technological University, \\ School of Electrical and Electronics Engineering, \\ Nanyang Avenue, Singapore 639798 \\ ${ }^{\mathrm{b}}$ Singapore Institute of Manufacturing Technology, \\ 71 Nanyang Avenue, Singapore 638075
}

\begin{abstract}
We present an all-fiber linear-phase equalizer for equalizing Gaussian-like spectra. The equalizer uses an optical lattice filter in a symmetric folded structure. Compared to existing solutions to linear phase equalization, the proposed equalizer has guaranteed linear phase responses and lower power attenuation. As an illustrative example, the effectiveness of the proposed equalizer is demonstrated with the flattening of the output spectrum of a superluminescent light emitting diode. (๑) 2006 Society of Photo-Optical Instrumentation Engineers.
\end{abstract}

[DOI: 10.1117/1.2280239]

Subject terms: linear phase; Gaussian-like spectra; optical lattice filter; superluminescent light-emitting diode; maximally flat spectrum; equalization.

Paper 060242LR received Apr. 3, 2006; revised manuscript received Jun. 19, 2006; accepted for publication Jun. 23, 2006; published online Aug. 16, 2006.

\section{Introduction}

In long-haul optical communications and optical engineering related applications, many components/devices have Gaussian-like spectral responses, and these spectra must be equalized to attain certain optical processing properties. The equalization filter is not only required to yield the maximal flattness and minimal attenuation loss in the spectral amplitude response, it is also desired to have a linear phase response to eliminate signal distortion resulting from dispersion. ${ }^{1}$ For example, in the application of supercontinuum (SC) pulse sources for optical time-division multiplexing (OTDM) and wavelength-division multiplexing ${ }^{2}$ (WDM), the SC spectra that generally have Gaussian shapes must be very flat for channel power equalization so as to apply the spectrum splicing technique. In addition, the phase distortion on the equalization of the coherent SC sources should be as small as possible due to the stringent dispersion requirements of those OTDM and WDM optical systems. ${ }^{1}$

Recently, several spectrum equalization schemes have been proposed to equalize Gaussian-like spectra by using an all-fiber optical lattice filter that has either a forward structure $^{3}$ or a feedback structure. ${ }^{4}$ The designed equalization filters were demonstrated to be capable of flattening the Gaussian-like spectra in the maximally flat sense with minimal attenuation loss. However, because the phase responses of the equalization filters were not considered in the filter design, these filters tended to have nonlinear phases. In this letter, we demonstrate a novel all-fiber

0091-3286/2006/\$22.00 @ 2006 SPIE equalizer that has a symmetric folded lattice structure. Although a similar structure has been used in integrated optics $^{5}$ to achieve flat add-drop filters, its use in achieving linear phase has not been explored. We show that the linear-phase characteristics of the equalization filter with the symmetric folded structure is naturally guaranteed and that the desired amplitude responses can be obtained by the same approaches proposed in existing equalization schemes.

\section{Design of the Equalization Filter with Symmetric Folded Structure}

The proposed equalization filter consists of two optical lattice filters, which are denoted by lattice filter 1 and lattice filter 2, as shown in Fig. 1. Each optical lattice filter is formed by linking three directional couplers with differential time-delay lines. The two lattice filters have the same design parameters, and they are connected with a unit differential delay line in a symmetric folded manner.

To highlight the principle of the proposed equalization filter, we derive the spectral transmission of the filter. We first introduce the following notation. The power coupling ratios of the directional couplers are denoted by $\kappa_{i}(i$ $=1,2,3)$. They are the design parameters. The differential delay lines are selected to have a differential length $\Delta L$. They are chosen according to the band that the filter is intended. We denote the electric fields at the $i$ 'th port of lattice filter 1 and lattice filter 2 by $E_{i}^{\prime}$ and $E_{i}^{\prime \prime}(i$ $=1,2,3,4)$, respectively, as shown in Fig. 1 . We further denote the optical angular frequency as $w$, where $w$ $=\Delta L \pi n_{\text {ref }} / 2 \lambda, \lambda$ is the wavelength of the wave propagating through free space, and $n_{\text {ref }}$ is the refractive index of the optical fiber. The field transfer functions with respect to the bar-state (from $E_{1}^{\prime}$ to $E_{3}^{\prime}$ ) and the cross-state (from $E_{1}^{\prime}$ to $E_{4}^{\prime}$ ) of lattice filter 1 are functions of $w$ and denoted as $T_{\mathrm{bar}}(w)$ and $T_{\text {cross }}(w)$, respectively. Correspondingly, the bar-state and the cross-state transmissions are represented as $Q_{\text {bar }}(w)$ and $Q_{\text {cross }}(w)$, respectively, and the overall transmission of the proposed equalizer can be expressed by $P(w)$ $=\left|Q_{\text {cross }}(w)\right|^{2}$.

Using the elementary field transfer functions of the fiber couplers and the differential lines which are available in the literatures, ${ }^{3,4}$ we can easily obtain the expressions of the field transfer functions ${ }^{3} T_{\text {bar }}(w)$ and $T_{\text {cross }}(w)$. According to the proposed filter structure, $Q_{\text {cross }}(w)$ can be expressed in terms of the field transfer function as

$Q_{\text {cross }}(w)=T_{\text {bar }}^{*}(w) T_{\text {cross }}(w) e^{j w}-T_{\text {bar }}(w) T_{\text {cross }}^{*}(w) e^{-j w}$,

where the superscript ${ }^{*}$ represents the complex conjugate. Substituting $T_{\text {bar }}(w)$ and $T_{\text {cross }}(w)$ into $Q_{\text {cross }}(w)$, we have

$Q_{\text {cross }}(w)=-j\left(f_{1,6} \cos 5 w+f_{2,6} \cos 3 w+f_{3,6} \cos w\right)$,

where $j=\sqrt{-1}$ and $f_{i, 6}(i=1,2,3)$ are real constants determined by the fiber coupling ratios. From Eq. (1), it can be easily verified that the transmission transfer function $Q_{\text {cross }}(w)$ has a linear phase response as it consists of odd frequency components such as $\cos [2(k-1) w](k$ is an integer) elements. Thus, regardless of the fiber coupling ratios of the filter, the linear phase characteristics of the equaliza- 


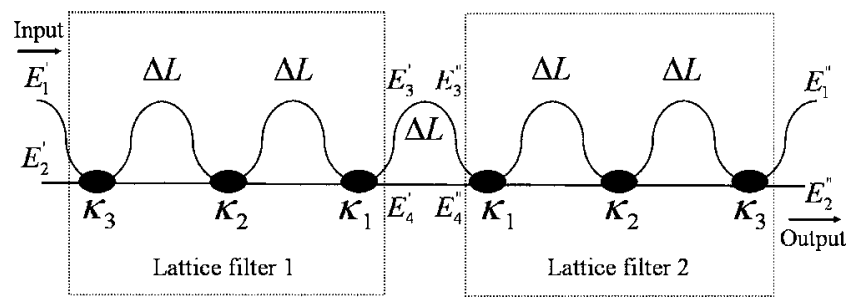

Fig. 1 Schematic of the spectral equalization filter with a symmetric folded structure.

tion filter is guaranteed automatically by the proposed structure. Then, the design task of equalization filter is reduced to the design of the amplitude response of $P(w)$ by choosing appropriate parameters $\kappa_{i}(i=1,2,3)$ to meet the requirements such as maximal flatness and minimal attenuation loss. This can be done by performing the same optimization procedures as in literature, ${ }^{3}$ where the transfer functions $Q_{\text {cross }}(w)$ and $Q_{\text {bar }}(w)$ are first solved by minimizing the attenuation loss subject to maximal flatness conditions, then the fiber coupling ratios are determined by a recursive algorithm. Clearly, the optimization procedures ${ }^{3}$ also allow for an easy extension of the proposed equalization filter to the general case where $n$ 'th order lattice filters are used in place of lattice filters 1 and 2 in Fig. 1. In this case, the higher the order of the lattice filter used, the flatter is the output spectrum, but at the expense of higher attenuation. A trade-off between the flatness of the transmission and the output power should therefore be taken into consideration in practice.

\section{Experimental Results}

As an illustrative example, we now consider the use of the proposed lattice equalizer in Fig. 1 for the equalization of an SLED transmission. Using the least-squares approximation method, the analytical Gaussian function is obtained as $F(w)=\beta \exp \left[-\left(w-w_{0}\right)^{2} / 2 \delta^{2}\right]$ with the central wavelength taking the value of $1544.2 \mathrm{~nm}$, the amplitude $\beta=0.98$, and the deviation $\delta=0.66$. The corresponding approximation error is within the range of $0.015 \mathrm{~dB}$. Follow the minimization procedures, ${ }^{3}$ the optimal coefficients of $Q_{\text {cross }}(w)$ are

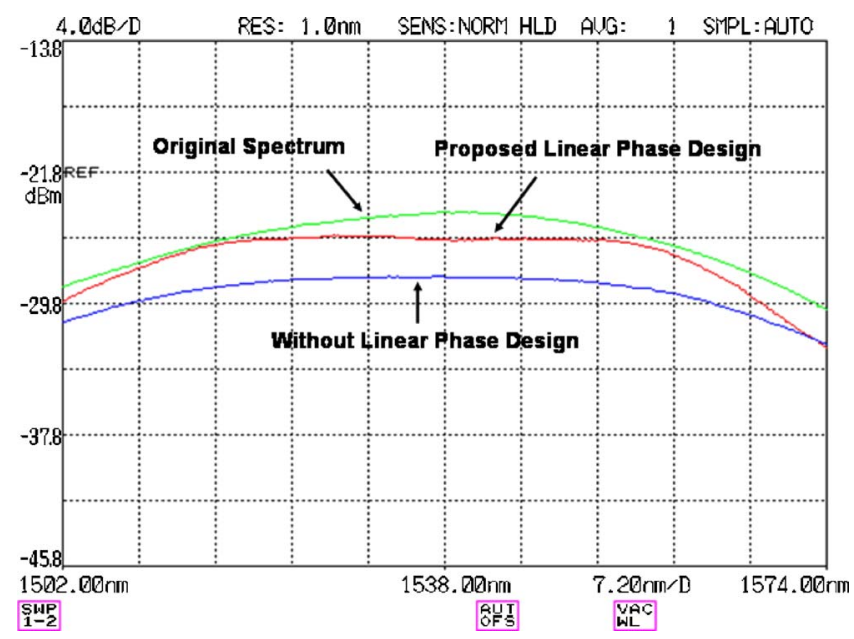

Fig. 2 Equalized spectra of a SLED.

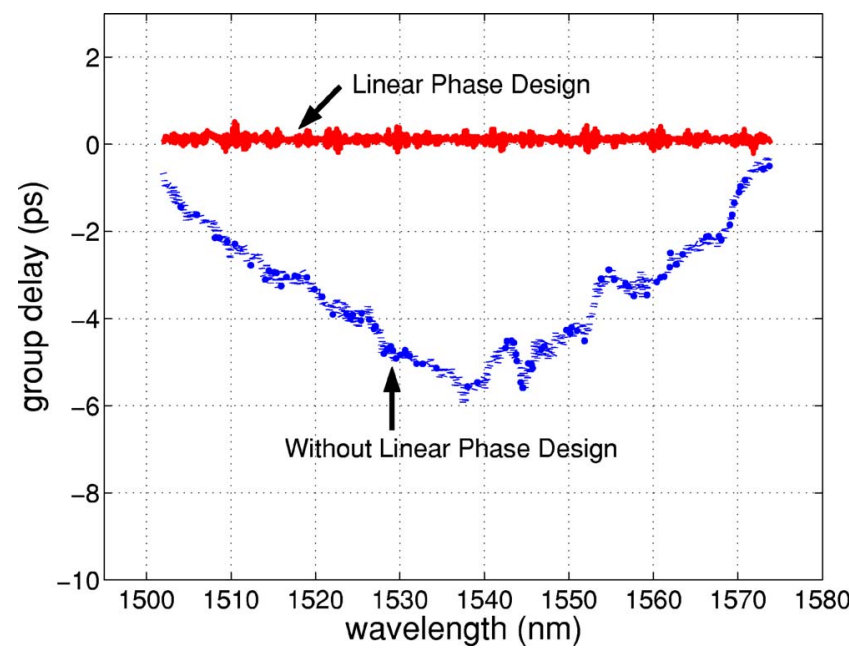

Fig. 3 Phase responses of spectrum equalization filters.

obtained as $f_{1,6}=0.0485, f_{2,6}=-0.3533$, and $f_{3,6}=1.1035$, and the required fiber coupling ratios are given by $\kappa_{1}$ $=9.93 \%, \kappa_{2}=79.12 \%$, and $\kappa_{3}=10.66 \%$, respectively.

We use these optimal values to fabricate the fiber lattice equalization filter. The fabrication is done by the reported approach, ${ }^{6}$ where fiber couplers are fabricated successively on two pieces of fibers without any splicing. To examine the equalization performance, we connect the filter to the output of the superluminescent light-emitting diode (SLED) where the Gaussian-like spectrum is measured. The equalized spectrum is observed on an optical spectrum analyzer and is shown in Fig. 2. The phase responses of the proposed equalizer is examined separately by measuring its group delay with an optical dispersion analyzer (Agilent 86038A). Figure 3 shows the measured phase responses. As a comparison with existing equalization filters, we consider an alternative equalization filter with the same number of fiber couplers but the two lattice filters are connected in a feedback structure, since this kind of equalization filter has been demonstrated to be capable of delivering lower attenuation loss. ${ }^{4}$ Using the feedback structure equalization filter, the equalized amplitude transmissions for the same SLED source is shown in Fig. 2 and the phase responses of the equalization filter are included in Fig. 3, respectively. Comparing the performance of the two filters, it is clear that both deliver a flat transmission with almost the same 0.5 and 3-dB passband bandwidths, but the proposed equalizer can deliver a constant group delay while the feedback structure equalization filter has an inevitable group delay variation of around $6 \mathrm{ps}$ in the passband transmission. In addition, the proposed equalization filter can improve the equalized output power by $2 \mathrm{~dB}$ per wavelength in the bandwidth. The experimental results demonstrate the effectiveness of the proposed design approach.

\section{Conclusion}

We presented a novel symmetric folded structure to realize an all-fiber equalization filter for Gaussian-like spectra. The proposed structure ensures the linear phase responses of the equalization filter regardless of the amplitude responses. Thus, the design of the equalization filter to satisfy both phase and amplitude requirements is simplified to one of 


\section{OE LETTERS}

designing for only the desired amplitude responses, and this problem has been well addressed in the existing literature. The proposed structure can also be easily extended to a higher order equalization filter design. Experimental results showed that the spectral equalization filter with the proposed structure can deliver the linear phase response while attaining comparable or better amplitude performance than that of existing spectral equalization filters.

\section{Acknowledgments}

This work is partially supported by CRP U04-P-106B, Research Grant RGM4/04, and the Singapore Millennium Foundation.

\section{References}

1. G. Lenz, B. J. Eggleton, C. K. Madsen, C. R. Giles, and G. Nykolak, "Optimal dispersion of optical filters for WDM systems," IEEE Photon. Technol. Lett. 10, 567-569 (1998).

2. Y. Takushima and K. Kikuchi, "10-GHz, over 20-channel multiwavelength pulse source by slicing super-continuum spectrum generated in normal-dispersion fiber," IEEE Photon. Technol. Lett. 11, 322-324 (1999).

3. Y. Zhang, Q. J. Wang, and Y. C. Soh, "Equalization of Gaussian-like spectra with optical lattice fitters," J. Opt. Soc. Am. B 22, 1498-1511 (2005).

4. Z. G. Dong, Y. Zhang, Q. J. Wang, and Y. C. Soh, "Gaussian-like spectrum equalization by using an all-fiber lattice filters with symmetric feedback structure," Opt. Eng. 44, 050504 (May 2005).

5. B. J. Offrein, F. Horst, G. L. Bona, H. W. Salemink, R. Germann, and R. Beyeler, "Wavelength tunable 1-from-16 and flat passband 1-from-8 add-drop filters," IEEE Photon. Technol. Lett. 11, 14401442 (Nov. 1999).

6. Q. J. Wang, Y. Zhang, and Y. C. Soh, "Design of spectrum equalization filter for SLED light source," Opt. Commun. 229, 223-231 (2003) 